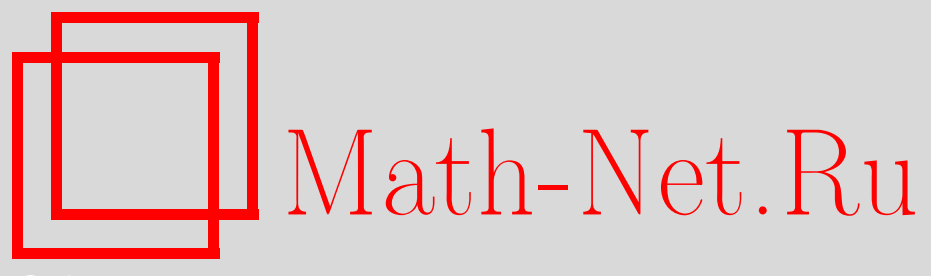

А. Е. Миронов, О полиномиальных интегралах механической системы на двумерном торе, Изв. РАН. Сер. матем., 2010, том 74, выпуск 4, 145-156

DOI: https://doi.org/10.4213/im3989

Использование Общероссийского математического портала Math-Net.Ru подразумевает, что вы прочитали и согласны с пользовательским соглашением http://www . mathnet.ru/rus/agreement

Параметры загрузки:

IP : 3.89 .197 .203

26 апреля 2023 г., 13:24:33

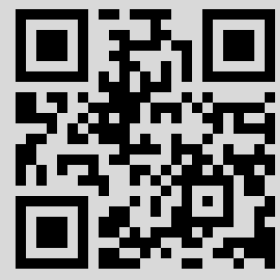


УДК 517.938

\author{
А. Е. Миронов
}

\title{
О полиномиальных интегралах механической системы на двумерном торе
}

\begin{abstract}
Показано, что если натуральная механическая система на двумерном торе с вещественным аналитическим потенциалом обладает полиномиальным интегралом нечетной степени по импульсам, то старшие коэффициенты при импульсах удовлетворяют двум тождествам специального вида. Также показано, что если система обладает интегралом пятой степени по импульсам, то существует интеграл первой степени по импульсам.

Библиография: 6 наименований.
\end{abstract}

Ключевые слова: интегрируемые гамильтоновы системы, полиномиальные интегралы.

\section{§ 1. Введение}

В настоящей работе мы докажем, что если гамильтонова система

$$
\dot{x}=\frac{\partial H}{\partial p_{1}}, \quad \dot{y}=\frac{\partial H}{\partial p_{2}}, \quad \dot{p}_{1}=-\frac{\partial H}{\partial x}, \quad \dot{p}_{2}=-\frac{\partial H}{\partial y}
$$

с гамильтонианом

$$
H=\frac{p_{1}^{2}+p_{2}^{2}}{2}+V(x, y)
$$

где $V$ - вещественная аналитическая периодическая функция на плоскости $\mathbb{R}^{2}$ с некоторой решеткой периодов $\Lambda \subset \mathbb{R}^{2}$, имеет первый интеграл нечетной степени по импульсам, который не сводится к интегралу первой степени, то коэффициенты при старших степенях импульсов удовлетворяют двум линейным тождествам специального вида. В работе также доказано, что если $V$ - вещественная аналитическая периодическая функция по переменным $x, y$ и если система (1.1) имеет интеграл пятой степени по импульсам, то существует интеграл первой степени по импульсам.

Несложно указать потенциалы, для которых система (1.1) имеет интегралы первой или второй степени. А именно, если потенциал имеет вид

$$
V=V\left(a_{1} x+b_{1} y\right)
$$

либо вид

$$
V=V_{1}\left(a_{2} x+b_{2} y\right)+V_{2}\left(a_{3} x+b_{3} y\right),
$$

то гамильтонова система (1.1) может иметь соответственно интеграл первой и второй степени по импульсам (константы $a_{j}, b_{j}$ должны быть согласованы специальным образом с решеткой $\Lambda$ ).

Работа выполнена при финансовой поддержке РФФИ (грант № 06-01-00094а), Программы фундаментальных исследований РАН "Фундаментальные проблемы нелинейной динамики", а также Программы Президента РФ "Поддержка молодых кандидатов" (грант MK-5430.2008.1).

(C) А. Е. Миронов, 2010 
Вопрос о существовании у системы (1.1) полиномиальных интегралов более высокой степени является до сих пор нерешенным. Известны следующие частные результаты. М.Л. Бялый [1] показал, что если потенциал $V$ периодичен по $x$ и $y$ и существует интеграл третьей степени по импульсам, то существует интеграл первой степени по импульсам. Н. В. Денисова и В. В. Козлов [2] обобщили этот результат на произвольную решетку периодов $\Lambda \subset \mathbb{R}^{2}$ функции $V$. В [1] анонсирована теорема об интегралах четвертой степени. В. В. Козловым и Д.В. Трещевым [3] доказано, что если потенциал $V$ является тригонометрическим полиномом и существует полиномиальный интеграл, то существует интеграл первой или второй степени.

Отметим, что если пространство положений является ориентируемой поверхностью $\Sigma$ рода больше 1 , а гамильтониан является аналитической функцией на кокасательном расслоении к $\Sigma$ :

$$
H: T^{*} \Sigma \rightarrow \mathbb{R}
$$

где $H=T(p, q)+V(q), T(p, q)$ при фиксированном $q$ является положительно определенной квадратичной формой на $T_{q}^{*} \Sigma$, то гамильтонова система $\dot{q}=\frac{\partial H}{\partial p}$, $\dot{p}=-\frac{\partial H}{\partial q}$ по теореме Козлова [4] не может иметь аналитического первого интеграла. Если же пространство положений $\Sigma$ является двумерной сферой, то указанная гамильтонова система может иметь интегралы более высокой степени, чем 2.

Наличие у гамильтоновой системы (1.1) интеграла $F=F\left(x, y, p_{1}, p_{2}\right)$, постоянного на траекториях, эквивалентно равенству нулю скобки Пуассона от $H$ и $F$ :

$$
\{H, F\}=\left(\frac{\partial H}{\partial p_{1}} \frac{\partial F}{\partial x}-\frac{\partial H}{\partial x} \frac{\partial F}{\partial p_{1}}\right)+\left(\frac{\partial H}{\partial p_{2}} \frac{\partial F}{\partial y}-\frac{\partial H}{\partial y} \frac{\partial F}{\partial p_{2}}\right)=0 .
$$

Предположим, что существует полиномиальный интеграл по импульсам

$$
F=F_{n}+\cdots+F_{0},
$$

где $F_{k}$ - однородный полином по $p_{1}$ и $p_{2}$ степени $k$,

$$
F_{k}=\sum_{j=0}^{k} b_{j}^{k}(x, y) p_{1}^{k-j} p_{2}^{j}
$$

Как показал С. В. Болотин [5], если $V$ - аналитическая функция и $b_{j}^{k} \in C^{1}$, то из условия $\{H, F\}=0$ следует, что $b_{j}^{k}$ - аналитические функции. Поэтому мы изначально будем предполагать, что коэффициенты $b_{j}^{k}$ аналитические.

Заметим, что скобка Пуассона от $H$ и однородного полинома $F_{k}$ содержит только мономы одинаковой четности, поэтому изучение вопроса о существовании полиномиального интеграла сводится к рассмотрению двух случаев: когда все $F_{k}$ либо четной степени, либо нечетной степени по $p_{1}$ и $p_{2}$. В этой работе мы рассматриваем случай, когда

$$
F=F_{2 n+1}+F_{2 n-1}+\cdots+F_{1} .
$$

Можно показать, что старшие коэффициенты $b_{j}^{2 n+1}$ являются константами [1]. Эти константы мы будем обозначать через

$$
a_{j}=b_{j}^{2 n+1} .
$$


Введем следующие обозначения:

$$
\begin{gathered}
\alpha=(2 n+1) ! ! \cdot a_{0}+(2 n-1) ! ! \cdot 1 ! ! \cdot a_{2}+(2 n-3) ! ! \cdot 3 ! ! \cdot a_{4}+\cdots \\
\quad \cdots+1 ! ! \cdot(2 n-1) ! ! \cdot a_{2 n}, \\
\beta=(2 n+1) ! ! \cdot a_{2 n+1}+(2 n-1) ! ! \cdot 1 ! ! \cdot a_{2 n-1}+(2 n-3) ! ! \cdot 3 ! ! \cdot a_{2 n-3}+\cdots \\
\quad \cdots+1 ! ! \cdot(2 n-1) ! ! \cdot a_{1},
\end{gathered}
$$

где $(2 k+1) ! !=1 \cdot 3 \cdots(2 k+1)$.

Основными результатами этой работы являются следующие теоремы.

Теорема 1.1. Предположим, что гамилътонова система (1.1) имеет полиномиалъный первый интеграл нечетной степени по импульсам. Тогда либо $\alpha=0$ и $\beta=0$, либо существует интеграл первой степени по импульсам.

Теорема 1.2. Предположим, что потенциал $V$ периодичен по х и $y$ :

$$
V(x+1, y)=V(x, y+1)=V(x, y) .
$$

Предположим также, что гамильтонова система (1.1) имеет первый интеграл пятой степени по импульсам. Тогда существует интеграл первой степени по импульсам.

Основная идея доказательства теоремы 1.1 заключается в следующем. Из условия равенства нулю скобки Пуассона $\{H, F\}$ мы покажем, что существует $\Lambda$-периодическая функция $\mathcal{F}$ со специальными свойствами (см. ниже), которая удовлетворяет уравнению

$$
\mathcal{F}_{y} V_{x}-\mathcal{F}_{x} V_{y}+\alpha V_{x}+\beta V_{y}=0 .
$$

В рассматриваемом (вещественном аналитическом) случае будет показано, что решения этого уравнения имеют вид

$$
\mathcal{F}=G(V)+\beta x-\alpha y,
$$

где $G$ - функция одного переменного. Если система не имеет интеграла первой степени по импульсам, то $\mathcal{F}$ будет $\Lambda$-периодической функцией только в случае $\alpha=\beta=0$.

В $\S 2$ мы докажем теорему 1.1 , а в $\S 3$ - теорему 1.2 .

Автор выражает благодарность М. Л. Бялому за внимание к этой работе и за приглашение посетить Университет Тель-Авива. Автор благодарен этому университету за теплое гостеприимство.

\section{§ 2. Доказательство теоремы 1.1}

2.1. Вывод уравнения (1.2). Условие равенства нулю скобки Пуассона $\{H, F\}=0$ эквивалентно системе нелинейных уравнений для коэффициентов $b_{j}^{k}$. Заметим, что если $F-$ полиномиальная функция указанного выше вида, то скобка Пуассона с $H$ имеет вид

$$
\{H, F\}=G_{2 n}+G_{2(n-1)}+\cdots+G_{0},
$$

где $G_{k}$ - однородный полином степени $k$. Поэтому данная система разбивается на группы из $n+1$ уравнений. В первую группу входит одно уравнение:

$$
L_{\{1,1\}} \equiv b_{0}^{1} V_{x}+b_{1}^{1} V_{y}=0 .
$$

Это уравнение эквивалентно тому, что $G_{0}=0$. 
Ко второй группе относятся три уравнения:

$$
\begin{gathered}
L_{\{2,1\}} \equiv b_{0_{x}}^{1}=3 b_{0}^{3} V_{x}+b_{1}^{3} V_{y}, \\
b_{0_{y}}^{1}+b_{1_{x}}^{1}=2 b_{1}^{3} V_{x}+2 b_{2}^{3} V_{y}, \\
L_{\{2,2\}} \equiv b_{1_{y}}^{1}=b_{2}^{3} V_{x}+3 b_{3}^{3} V_{y} .
\end{gathered}
$$

Эти уравнения эквивалентны тому, что коэффициенты при $p_{1}^{2}, p_{1} p_{2}$ и $p_{2}^{2}$ в $\{H, F\}$ равны нулю.

Третью группу составляют следующие уравнения:

$$
\begin{gathered}
L_{\{3,1\}} \equiv b_{0_{x}}^{3}=5 b_{0}^{5} V_{x}+b_{1}^{5} V_{y}, \\
b_{0_{y}}^{3}+b_{1_{x}}^{3}=4 b_{1}^{5} V_{x}+2 b_{2}^{5} V_{y}, \\
L_{\{3,2\}} \equiv b_{1_{y}}^{3}+b_{2_{x}}^{3}=3 b_{2}^{5} V_{x}+3 b_{3}^{5} V_{y}, \\
b_{2_{y}}^{3}+b_{3_{x}}^{3}=2 b_{3}^{5} V_{x}+4 b_{4}^{5} V_{y}, \\
L_{\{3,3\}} \equiv b_{3_{y}}^{3}=b_{4}^{5} V_{x}+5 b_{5}^{5} V_{y} .
\end{gathered}
$$

В $k$-ю группу входят $(2 k-1)$ уравнений:

$$
\begin{gathered}
L_{\{k, 1\}} \equiv b_{0_{x}}^{2 k-1}=(2 k+1) b_{0}^{2 k+1} V_{x}+b_{1}^{2 k+1} V_{y}, \\
b_{0_{y}}^{2 k-1}+b_{1_{x}}^{2 k-1}=(2 k) b_{1}^{2 k+1} V_{x}+2 b_{2}^{2 k+1} V_{y}, \\
L_{\{k, 2\}} \equiv b_{1_{y}}^{2 k-1}+b_{2_{x}}^{2 k-1}=(2 k-1) b_{2}^{2 k+1} V_{x}+3 b_{3}^{2 k+1} V_{y}, \\
\ldots \ldots \ldots \cdots \cdots \cdots \cdots \cdots \cdots \cdots \cdots \cdots \cdots \cdots \cdots \cdots \cdots \cdots \cdots \cdots \cdots \cdots \cdots \cdots \cdots \cdots \cdots \cdots+b_{2 k-1}^{2 k+1} V_{y}, \\
L_{\{k, k-1\}} \equiv b_{2 k-3_{y}}^{2 k-1}+b_{2 k-2_{x}}^{2 k-1}=3 b_{2 k-2}^{2 k+1} V_{x}+(2 k-1) \\
b_{2 k-2_{y}}^{2 k-1}+b_{2 k-1_{x}}^{2 k-1}=2 b_{2 k-1}^{2 k+1} V_{x}+(2 k) b_{2 k}^{2 k+1} V_{y}, \\
L_{\{k, k\}} \equiv b_{2 k-1_{y}}^{2 k-1}=b_{2 k}^{2 k+1} V_{x}+(2 k+1) b_{2 k+1}^{2 k+1} V_{y} .
\end{gathered}
$$

И наконец, в последнюю, $(n+1)$-ю группу, входят $(2 n+1)$ уравнений:

$$
\begin{gathered}
L_{\{n+1,1\}} \equiv b_{0_{x}}^{2 n-1}=(2 n+1) a_{0} V_{x}+a_{1} V_{y}, \\
b_{0_{y}}^{2 n-1}+b_{1_{x}}^{2 n-1}=(2 n) a_{1} V_{x}+2 a_{2} V_{y}, \\
L_{\{n+1,2\}} \equiv b_{1_{y}}^{2 k-1}+b_{2_{x}}^{2 k-1}=(2 n-1) a_{2} V_{x}+3 a_{3} V_{y}, \\
\ldots \ldots \ldots \ldots \ldots \ldots \ldots \ldots \ldots \ldots \ldots \ldots \ldots \ldots \ldots \ldots \ldots \ldots \ldots \ldots \ldots \\
L_{\{n+1, n\}} \equiv b_{2 n-3_{y}}^{2 n-1}+b_{2 n-2_{x}}^{2 n-1}=3 a_{2 n-2} V_{x}+(2 n-1) a_{2 n-1} V_{y}, \\
b_{2 n-2_{y}}^{2 n-1}+b_{2 n-1_{x}}^{2 n-1}=2 a_{2 n-1} V_{x}+(2 n) a_{2 n} V_{y}, \\
L_{\{n+1, n+1\}} \equiv b_{2 n-1_{y}}^{2 n-1}=a_{2 n} V_{x}+(2 n+1) a_{2 n+1} V_{y} .
\end{gathered}
$$

В каждой группе уравнений нас в дальнейшем будут интересовать не все уравнения, а только те, которые обозначены соответственно $L_{\{k, s\}}$.

Справедлива

ЛЕмма 2.1. Для любого $k=1, \ldots, n$ существует $\Lambda$-периодическая функиия $F^{2 k+1}$, которая удовлетворяет уравнению

$$
\begin{array}{r}
\left((2 k+1) ! ! \cdot b_{0}^{2 k+1}+(2 k-1) ! ! \cdot 1 ! ! \cdot b_{2}^{2 k+1}+(2 k-3) ! ! \cdot 3 ! ! \cdot b_{4}^{2 k+1}+\cdots\right. \\
\left.\cdots+1 ! ! \cdot(2 k-1) ! ! \cdot b_{2 n}^{2 k+1}+F_{y}^{2 k+1}\right) V_{x} \\
+\left((2 k+1) ! ! \cdot b_{2 k+1}^{2 k+1}+(2 k-1) ! ! \cdot 1 ! ! \cdot b_{2 k-1}^{2 k+1}+(2 k-3) ! ! \cdot 3 ! ! \cdot b_{2 k-3}^{2 k+1}+\cdots\right. \\
\left.\cdots+1 ! ! \cdot(2 k-1) ! ! \cdot b_{1}^{2 k+1}-F_{x}^{2 k+1}\right) V_{y}=0
\end{array}
$$


u которая имеет вид

$$
F^{2 k+1}=\frac{F_{1}^{2 k+1}}{V_{x}^{k}}=\frac{F_{2}^{2 k+1}}{V_{y}^{k}},
$$

где $F_{1}^{2 k+1}, F_{2}^{2 k+1}$ - аналитические $\Lambda$-периодические функции на плоскости $\mathbb{R}^{2}$.

Таким образом, если функция $F^{2 k+1}$ имеет особенности, то только в тех точках, в которых $V_{x}=V_{y}=0$.

ДоКАЗАТЕЛЬСТво ЛЕммы 2.1 будем проводить по индукции. Обозначим через $F^{3}$ функцию $-b_{1}^{1} / V_{x}$. Тогда из уравнения $L_{\{1,1\}}=0$ имеем

$$
F^{3}=-\frac{b_{1}^{1}}{V_{x}}=\frac{b_{0}^{1}}{V_{y}},
$$

$F_{1}^{3}$ и $F_{2}^{3}$ равны соответственно $-b_{1}^{1}$ и $b_{0}^{1}$. Теперь сложим уравнения $L_{\{2,1\}}=0$ и $L_{\{2,2\}}=0$. Получим

$$
b_{0_{x}}^{1}+b_{1_{y}}^{1}=\left(3 b_{0}^{3}+b_{2}^{3}\right) V_{x}+\left(b_{1}^{3}+3 b_{3}^{3}\right) V_{y} .
$$

Подставим в левую часть последнего равенства выражения

$$
b_{0}^{1}=F^{3} V_{y}, \quad b_{1}^{1}=-F^{3} V_{x} .
$$

Получим

$$
\left(3 b_{0}^{3}+b_{2}^{3}+F_{y}^{3}\right) V_{x}+\left(3 b_{3}^{3}+b_{1}^{3}-F_{x}^{3}\right) V_{y}=0 .
$$

Таким образом, первый шаг индукции установлен.

Для большей ясности мы приведем сначала доказательство утверждения леммы при $k=2$, а затем объясним индукционный переход в общем случае.

В силу последнего уравнения введем функцию

$$
F^{5}=-\frac{3 b_{3}^{3}+b_{1}^{3}-F_{x}^{3}}{V_{x}}=\frac{3 b_{0}^{3}+b_{2}^{3}+F_{y}^{3}}{V_{y}} .
$$

Умножим обе части уравнений $L_{\{3,1\}}=0$ и $L_{\{3,3\}}=0$ на 3 , а затем сложим полученные уравнения и $L_{\{3,2\}}=0$. В результате получим

$$
\left(3 b_{0_{x}}^{3}+b_{2_{x}}^{3}\right)+\left(3 b_{1_{y}}^{3}+b_{1_{y}}^{3}\right)=\left(15 b_{0}^{5}+3 b_{2}^{5}+3 b_{4}^{5}\right) V_{x}+\left(15 b_{5}^{5}+3 b_{3}^{5}+3 b_{1}^{5}\right) V_{y} .
$$

С учетом (2.1) имеем

$$
\left(3 b_{0_{x}}^{3}+b_{2_{x}}^{3}+F_{x y}^{3}\right)+\left(3 b_{1_{y}}^{3}+b_{1_{y}}^{3}-F_{x y}^{3}\right)=F_{x}^{5} V_{y}-F_{y}^{5} V_{x} .
$$

Следовательно, справедливо равенство

$$
\left(15 b_{0}^{5}+3 b_{2}^{5}+3 b_{4}^{5}+F_{y}^{5}\right) V_{x}+\left(15 b_{5}^{5}+3 b_{3}^{5}+3 b_{1}^{5}-F_{x}^{5}\right) V_{y}=0 .
$$

Таким образом, лемма доказана при $k=2$.

Перейдем к общему случаю. Предположим, что лемма доказана при $k=$ $s-1$.

Умножим обе части уравнения $L_{\{s, 1\}}=0$ на $(2 s-1) !$ !, обе части $L_{\{s, 2\}}=0$ на $(2 s-3) ! ! \cdot 1 ! !, L_{\{s, 3\}}=0$ на $(2 s-5) ! ! \cdot 3 ! !, \ldots, L_{\{s, s-1\}}=0$ на $1 ! ! \cdot(2 s-3) ! !$, 
$L_{\{s, s\}}=0$ на $(2 s-1) ! !$ и сложим. Получим

$$
\begin{gathered}
\left((2 s-1) ! ! \cdot b_{0_{x}}^{2 s-1}+(2 s-3) ! ! \cdot 1 ! ! \cdot b_{2_{x}}^{2 s-1}+(2 s-5) ! ! \cdot 3 ! ! \cdot b_{4_{x}}^{2 s-1}+\cdots\right. \\
\left.\quad \cdots+1 ! ! \cdot(2 s-3) ! ! \cdot b_{2 s-2_{x}}^{2 s-1}\right)+\left((2 s-1) ! ! \cdot b_{2 s-1_{y}}^{2 s-1}+(2 s-3) ! ! \cdot 1 ! ! \cdot b_{2 s-3}^{2 s-1}\right. \\
\left.+(2 s-5) ! ! \cdot 3 ! ! \cdot b_{2 s-5}^{2 s-1}+\cdots+1 ! ! \cdot(2 s-3) ! ! \cdot b_{1_{y}}^{2 s-1}\right) \\
=\left((2 s+1)(2 s-1) ! ! \cdot b_{0}^{2 s+1}+(2 s-1)(2 s-3) ! ! \cdot 1 ! ! \cdot b_{2}^{2 s+1}\right. \\
+(2 s-3)(2 s-5) ! ! \cdot 3 ! ! \cdot b_{4}^{2 s+1}+\cdots \\
\left.\quad \cdots+3 \cdot 1 ! ! \cdot(2 s-3) ! ! \cdot b_{2 s-2}^{2 s+1}+(2 s-1) ! ! \cdot b_{2 s}^{2 s+1}\right) V_{x} \\
+\left((2 s+1)(2 s-1) ! ! \cdot b_{2 s+1}^{2 s+1}+(2 s-1)(2 s-3) ! ! \cdot 1 ! ! \cdot b_{2 s-1}^{2 s+1}\right. \\
+(2 s-3)(2 s-5) ! ! \cdot 3 ! ! \cdot b_{2 s-3}^{2 s+1}+\cdots \\
\left.\quad \cdots+3 \cdot 1 ! ! \cdot(2 s-3) ! ! \cdot b_{3}^{2 s+1}+(2 s-1) ! ! \cdot b_{1}^{2 s+1}\right) V_{y},
\end{gathered}
$$

или, эквивалентно,

$$
\begin{aligned}
& \left((2 s-1) ! ! \cdot b_{0_{x}}^{2 s-1}+(2 s-3) ! ! \cdot 1 ! ! \cdot b_{2_{x}}^{2 s-1}+(2 s-5) ! ! \cdot 3 ! ! \cdot b_{4_{x}}^{2 s-1}+\cdots\right. \\
& \left.\cdots+1 ! ! \cdot(2 s-3) ! ! \cdot b_{2 s-2_{x}}^{2 s-1}+F_{x y}^{2 s-1}\right) \\
& +\left((2 s-1) ! ! \cdot b_{2 s-1_{y}}^{2 s-1}+(2 s-3) ! ! \cdot 1 ! ! \cdot b_{2 s-3_{y}}^{2 s-1}+(2 s-5) ! ! \cdot 3 ! ! \cdot b_{2 s-5_{y}}^{2 s-1}+\cdots\right. \\
& \left.\cdots+1 ! ! \cdot(2 s-3) ! ! \cdot b_{1_{y}}^{2 s-1}-F_{x y}^{2 s-1}\right) \\
& =\left((2 s+1)(2 s-1) ! ! \cdot b_{0}^{2 s+1}\right. \\
& +(2 s-1)(2 s-3) ! ! \cdot 1 ! ! \cdot b_{2}^{2 s+1}+(2 s-3)(2 s-5) ! ! \cdot 3 ! ! \cdot b_{4}^{2 s+1}+\cdots \\
& \left.\cdots+3 \cdot 1 ! ! \cdot(2 s-3) ! ! \cdot b_{2 s-2}^{2 s+1}+(2 s-1) ! ! \cdot b_{2 s}^{2 s+1}\right) V_{x} \\
& +\left((2 s+1)(2 s-1) ! ! \cdot b_{2 s+1}^{2 s+1}+(2 s-1)(2 s-3) ! ! \cdot 1 ! ! \cdot b_{2 s-1}^{2 s+1}\right. \\
& +(2 s-3)(2 s-5) ! ! \cdot 3 ! ! \cdot b_{2 s-3}^{2 s+1}+\cdots \\
& \left.\cdots+3 \cdot 1 ! ! \cdot(2 s-3) ! ! \cdot b_{3}^{2 s+1}+(2 s-1) ! ! \cdot b_{1}^{2 s+1}\right) V_{y} .
\end{aligned}
$$

Теперь воспользуемся тем, что утверждение леммы доказано при $k=s-1$. Это позволяет ввести функцию $F^{s+1}$ из совместных равенств

$$
\begin{gathered}
\left((2 s-1) ! ! \cdot b_{0}^{2 s-1}+(2 s-3) ! ! \cdot 1 ! ! \cdot b_{2}^{2 s-1}+(2 s-5) ! ! \cdot 3 ! ! \cdot b_{4}^{2 s-1}+\cdots\right. \\
\left.\cdots+1 ! ! \cdot(2 s-3) ! ! \cdot b_{2 s-2}^{2 s-1}+F_{y}^{2 s-1}\right)=F^{2 s+1} V_{y}, \\
\left((2 s-1) ! ! \cdot b_{2 s-1}^{2 s-1}+(2 s-3) ! ! \cdot 1 ! ! \cdot b_{2 s-3}^{2 s-1}+(2 s-5) ! ! \cdot 3 ! ! \cdot b_{2 s-5}^{2 s-1}+\cdots\right. \\
\left.\cdots+1 ! ! \cdot(2 s-3) ! ! \cdot b_{1}^{2 s-1}-F_{x}^{2 s-1}\right)=-F^{2 s+1} V_{x} .
\end{gathered}
$$

Следовательно, равенство (2.2) имеет вид

$$
\begin{aligned}
F_{x}^{2 s+1} V_{y}-F_{y}^{2 s+1} V_{x}= & \left((2 s+1)(2 s-1) ! ! \cdot b_{0}^{2 s+1}+(2 s-1)(2 s-3) ! ! \cdot 1 ! ! \cdot b_{2}^{2 s+1}\right. \\
& +(2 s-3)(2 s-5) ! ! \cdot 3 ! ! \cdot b_{4}^{2 s+1}+\cdots \\
& \left.\quad \cdots+3 \cdot 1 ! ! \cdot(2 s-3) ! ! \cdot b_{2 s-2}^{2 s+1}+(2 s-1) ! ! \cdot b_{2 s}^{2 s+1}\right) V_{x} \\
& +\left((2 s+1)(2 s-1) ! ! \cdot b_{2 s+1}^{2 s+1}+(2 s-1)(2 s-3) ! ! \cdot 1 ! ! \cdot b_{2 s-1}^{2 s+1}\right. \\
& +(2 s-3)(2 s-5) ! ! \cdot 3 ! ! \cdot b_{2 s-3}^{2 s+1}+\cdots \\
& \left.\quad \cdots+3 \cdot 1 ! ! \cdot(2 s-3) ! ! \cdot b_{3}^{2 s+1}+(2 s-1) ! ! \cdot b_{1}^{2 s+1}\right) V_{y},
\end{aligned}
$$


или, равносильно,

$$
\begin{aligned}
F_{x}^{2 s+1} V_{y}-F_{y}^{2 s+1} V_{x}=( & (2 s+1) ! ! \cdot b_{0}^{2 s+1}+(2 s-1) ! ! \cdot 1 ! ! \cdot b_{2}^{2 s+1} \\
& \left.+(2 s-3) ! ! \cdot 3 ! ! \cdot b_{4}^{2 s+1}+\cdots+1 ! ! \cdot(2 s-1) ! ! \cdot b_{2 n}^{2 s+1}\right) V_{x} \\
& +\left((2 s+1) ! ! \cdot b_{2 s+1}^{2 s+1}+(2 s-1) ! ! \cdot 1 ! ! \cdot b_{2 s-1}^{2 s+1}\right. \\
& \left.+(2 s-3) ! ! \cdot 3 ! ! \cdot b_{2 s-3}^{2 s+1}+\cdots+1 ! ! \cdot(2 s-1) ! ! \cdot b_{1}^{2 s+1}\right) V_{y} .
\end{aligned}
$$

Лемма доказана.

При $k=n$ имеем

СлЕДСТвиЕ 2.1. Имеет место равенство $F_{x}^{2 n+1} V_{y}-F_{y}^{2 n+1} V_{x}=\alpha V_{x}+\beta V_{y}$.

2.2. Общее аналитическое решение уравнения (1.2). В п. 2.1 мы установили (следствие 2.1), что если система (1.1) имеет полиномиальный интеграл нечетной степени по импульсам, то уравнение (1.2) обладает решением со следующими свойствами:

1) решение $\mathcal{F}$ является $\Lambda$-периодичным;

2) на множестве $\mathbb{R}^{2} \backslash\left\{V_{x}=0, V_{y}=0\right\}$ решение $\mathcal{F}$ является вещественным аналитическим;

3) функции $\mathcal{F} V_{x}^{n}$ и $\mathcal{F} V_{y}^{n}$ вещественные аналитические на всей плоскости $\mathbb{R}^{2}$.

Интуитивно ясно, что общее решение уравнения (1.2) имеет вид

$$
\mathcal{F}=G(V)+\beta x-\alpha y .
$$

Отсюда следует, что если функция $V$ существенным образом зависит от двух переменных $x$ и $y$, то функция $\mathcal{F}$ будет $\Lambda$-периодичной только в случае $\alpha=$ $\beta=0$. Дадим строгое обоснование этому утверждению.

Если потенциал имеет вид $V=V(a x+b y)$, то, как уже отмечалось, гамильтонова система (1.1) имеет интеграл первой степени по импульсам. В дальнейшем будем предполагать, что $V \neq V(a x+b y)$.

Любое решение уравнения (1.2) представимо в виде

$$
\mathcal{F}=W+\beta x-\alpha y
$$

где $W$ - некоторое решение однородного уравнения

$$
W_{x} V_{y}-W_{y} V_{x}=0 .
$$

Нам удобнее будет исследовать однородное уравнение. Геометрически уравнение $(2.3)$ означает, что якобиан отображения $(W, V): \mathbb{R}^{2} \rightarrow \mathbb{R}^{2}$ вырожден. По лемме 2.1 функция $W$ представима в виде

$$
W=\frac{f}{V_{x}^{n}},
$$

где $f$ - вещественная аналитическая функция на всей плоскости.

Выберем какую-то точку $p$ на плоскости, в которой $V_{x} \neq 0$. Пусть $\lambda$ - ненулевой элемент решетки $\Lambda$. Мы хотим сравнить значения функции $f$ в точках $p$ и $p+\lambda$. Справедлива

Лемма 2.2. Имеет место равенство $f(p)=f(p+\lambda)$. 
ДокАЗАтеЛЬСтво. Рассмотрим достаточно малую окрестность точки $p$, в которой $V_{x} \neq 0$. Любое решение уравнения $(2.3)$ в такой окрестности постоянно вдоль характеристик

$$
\dot{x}=V_{y}, \quad \dot{y}=-V_{x} .
$$

Характеристики уравнения (2.3) - кривые уровня функции $V$. Поэтому любое аналитическое решение в этой окрестности представимо в виде $W=G(V)$, где $G$ - аналитическая функция одной переменной. Таким образом, в малой окрестности точки $p$ функция $f$ имеет вид

$$
f=G_{p}(V) V_{x}^{n} .
$$

Аналогично, в малой окрестности точки $p+\lambda$ функция $f$ имеет вид

$$
f=G_{p+\lambda}(V) V_{x}^{n} .
$$

Соединим точки $p$ и $p+\lambda$ гладким путем, который трансверсально пересекается с кривыми уровней функции $V$ и кривой, заданной уравнением $V_{x}=0$ (рис. 1).

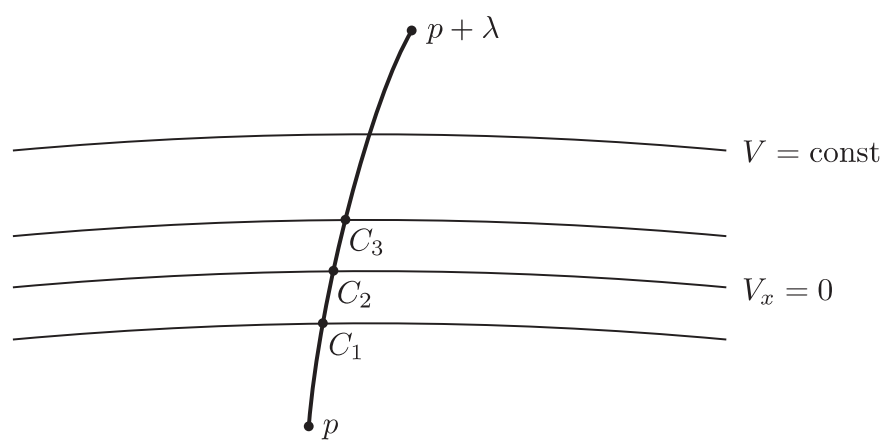

Рис. 1

Если этот путь не пересекается с кривой $V_{x}=0$, то в малой окрестности $U_{q}$ любой точки $q$ этого пути функция $f$ представима в виде

$$
f=G_{q}(V) V_{x}^{n}
$$

где $G_{q}$ - вещественная аналитическая функция одного аргумента. В силу однозначности аналитического продолжения в окрестности пути существует вещественная аналитическая функция $G$ такая, что

$$
f=G(V) V_{x}^{n},\left.\quad G(V)\right|_{U_{q}}=G_{q}(V),
$$

следовательно, функция $\mathcal{F}$ будет $\Lambda$-периодичной только в случае $\alpha=\beta=0$.

Если путь пересекается с кривой $V_{x}=0$, то функция $G_{p}(V)$ продолжаема только до точки пересечения, а в точке пересечения эта функция может иметь особенность, при переходе через которую функция $G_{p}$, например, может перейти на другую ветвь. Мы покажем, что на самом деле это невозможно.

Будем рассматривать $x$ и $y$ как комплексные координаты в $\mathbb{C}^{2}$. Продлим функцию $V$ до аналитической функции на окрестность вещественной плоскости $\mathbb{R}^{2} \subset \mathbb{C}^{2}$. Заметим, что множество нулей $V_{x}$ в $\mathbb{C}^{2}$ имеет вещественную 
коразмерность 2. Теперь мы изменим наш путь, соединяющий $p$ и $p+\lambda$ : при подходе к точке пересечения с нулями $V_{x}=0$ мы уйдем с вещественной плоскости $\mathbb{R}^{2} \subset \mathbb{C}^{2}$ и обойдем точку пересечения в $\mathbb{C}^{2}$, причем сделаем это так, чтобы $\operatorname{Im} V>0$. Это возможно, поскольку, как уже отмечалось, $\operatorname{codim}_{\mathbb{R}}\left\{V_{x}=0\right\}=2$.

В малой окрестности любой точки $q$ нового пути аналитическая функция $f$ представима в виде (2.4)

Отметим, что при движении по новому пути значения $V$ описывают стягиваемый замкнутый путь в комплексной плоскости, который не охватывает никаких особенностей.

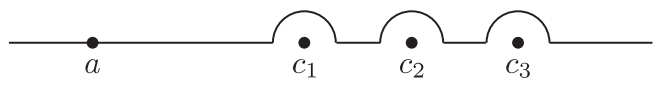

Рис. 2

На рис. 2 схематически изображены значения функции $V$ на комплексной плоскости вдоль нового пути, где $a=V(p)=V(p+\lambda)$, точки $c_{1}, c_{2}, c_{3}, \ldots$ отвечают точкам пересечения $C_{1}, C_{2}, C_{3}, \ldots$ первоначального пути с кривой $V_{x}=0$. Следовательно, в силу единственности аналитического продолжения имеем, что в окрестностях точек $p$ и $p+\lambda$ аналитическая функция $f$ представима в виде

$$
f=G(V) V_{x}^{n} .
$$

Из нашего предположения, что функция $V$ не является функцией вида $V=V(a x+b y)$, следует $f(p)=f(\lambda+p)$. Таким образом, функция $\mathcal{F}$ является $\Lambda$-периодичной только в случае $\alpha=\beta=0$. Лемма доказана. Это завершает доказательство теоремы 1.1.

\section{§ 3. Интегралы пятой степени}

В настоящем параграфе мы покажем, что в случае решетки $\Lambda=\mathbb{Z}^{2}$ если существует интеграл пятой степени по импульсам, то существует интеграл первой степени.

Предположим, что система (1.1) имеет интеграл пятой степени по импульсам

$$
\begin{aligned}
F=a_{0} p_{1}^{5}+ & a_{1} p_{1}^{4} p_{2}+a_{2} p_{1}^{3} p_{2}^{2}+a_{3} p_{1}^{2} p_{2}^{3}+a_{4} p_{1} p_{2}^{4}+a_{5} p_{2}^{5} \\
& +b_{0}^{3} p_{1}^{3}+b_{1}^{3} p_{1}^{2} p_{2}+b_{2}^{3} p_{1} p_{2}^{2}+b_{3}^{3} p_{2}^{3}+b_{0}^{1} p_{1}+b_{1}^{1} p_{2} .
\end{aligned}
$$

По теореме 1.1 старшие коэффициенты $a_{j}$ удовлетворяют двум тождествам

$$
a_{1}+a_{3}+5 a_{5}=0, \quad 5 a_{0}+a_{2}+a_{4}=0 .
$$

Разложим функцию $V$ в ряд Фурье:

$$
V=\sum_{\lambda^{*} \in \Lambda^{*}} v_{\lambda^{*}} \exp \left(2 \pi i\left(\lambda_{1}^{*} x+\lambda_{2}^{*} y\right)\right)
$$

где $\lambda^{*}=\left(\lambda_{1}^{*}, \lambda_{2}^{*}\right)$ - элемент решетки $\Lambda^{*}$, двойственной к решетке $\Lambda$ :

$$
\Lambda^{*}=\left\{\lambda^{*}:\left(\lambda^{*}, \lambda\right) \in \mathbb{Z}, \lambda \in \Lambda\right\} .
$$

Множество $S=\left\{\lambda^{*}: v_{\lambda^{*}} \neq 0\right\} \subset \Lambda$ называется спектром периодической функции $V$. 
Из уравнений

$$
\begin{gathered}
b_{0_{x}}^{3}=5 a_{0} V_{x}+a_{1} V_{y}, \\
b_{0_{y}}^{3}+b_{1_{x}}^{3}=4 a_{1} V_{x}+2 a_{2} V_{y}, \\
b_{1_{y}}^{3}+b_{2_{x}}^{3}=3 a_{2} V_{x}+3 a_{3} V_{y}, \\
b_{2_{y}}^{3}+b_{3_{x}}^{3}=2 a_{3} V_{x}+4 a_{4} V_{y}, \\
b_{3_{y}}^{3}=a_{4} V_{x}+5 a_{5} V_{y}
\end{gathered}
$$

исключим подходящим дифференцированием функции $b_{j}^{3}$. Получим

$$
\begin{aligned}
a_{4} \partial_{x}^{5} V & +\left(5 a_{5}-2 a_{3}\right) \partial_{x}^{4} \partial_{y} V+\left(3 a_{2}-4 a_{4}\right) \partial_{x}^{3} \partial_{y}^{2} V \\
& +\left(3 a_{3}-4 a_{1}\right) \partial_{x}^{2} \partial_{y}^{3} V+\left(5 a_{0}-2 a_{2}\right) \partial_{x} \partial_{y}^{4} V+a_{1} \partial_{y}^{5} V=0
\end{aligned}
$$

Отсюда следует, что спектр функции $V$ расположен на пяти прямых, проходящих через 0 (см. [1]), т. е. функция $V$ имеет вид

$$
V=\sum_{j=1}^{5} V_{j}\left(\alpha_{j} x+\beta_{j} y\right),
$$

где $V_{j}, j=1, \ldots, 5,-$ периодические функции, величины $z_{j}=\alpha_{j} / \beta_{j}$ удовлетворяют уравнению

$$
a_{4} z^{5}+\left(5 a_{5}-2 a_{3}\right) z^{4}+\left(3 a_{2}-4 a_{4}\right) z^{3}+\left(3 a_{3}-4 a_{1}\right) z^{2}+\left(5 a_{0}-2 a_{2}\right) z+a_{1}=0 .
$$

Обозначим через $F_{5}$ следующий полином:

$$
F_{5}=a_{0} p_{1}^{5}+a_{1} p_{1}^{4} p_{2}+a_{2} p_{1}^{3} p_{2}^{2}+a_{3} p_{1}^{2} p_{2}^{3}+a_{4} p_{1} p_{2}^{4}+a_{5} p_{2}^{5} .
$$

Уравнение (3.1) эквивалентно тому, что равенство

$$
p_{2} \frac{\partial F_{5}}{\partial p_{1}}-p_{1} \frac{\partial F_{5}}{\partial p_{2}}=0
$$

выполнено при $\alpha_{i} p_{1}+\beta_{i} p_{2}=0$, или, что то же самое, полином

$$
\alpha_{i} \frac{\partial F_{5}}{\partial p_{1}}+\beta_{i} \frac{\partial F_{5}}{\partial p_{2}}
$$

делится на $\alpha_{i} p_{1}+\beta_{i} p_{2}$. Обозначим через $Q$ частное от деления этих полиномов. Имеет место теорема, доказанная Н. В. Денисовой и В.В. Козловым [2]: на прямой $\alpha_{i} p_{1}+\beta_{i} p_{2}=0$ справедливо равенство

$$
\alpha_{i} \frac{\partial Q}{\partial p_{1}}+\beta_{i} \frac{\partial Q}{\partial p_{2}}=0 .
$$

Делая подходящий поворот в плоскости $x, y$, мы без ограничения общности можем считать, что одна из спектральных прямых вертикальна, т. е. $\alpha_{1}=0$. Тогда из (3.1) следует, что $a_{1}=0$, а условие (3.2) означает, что $a_{3}=0$.

Если спектр лежит на двух прямых, то либо вообще нет дополнительных интегралов, либо существует интеграл второй степени [6]. Поэтому мы будем считать, что $a_{1}=0, a_{3}=0$ и уравнения (3.1) и (3.2) имеют два различных непропорциональных решения $\alpha_{2}, \beta_{2}$ и $\alpha_{3}, \beta_{3}$. 
Сделаем поворот в плоскости $x, y$. Этот поворот индуцирует замену импульсов

$$
\begin{gathered}
p_{1}=P_{1} \cos \alpha+P_{2} \sin \alpha, \\
p_{2}=-P_{1} \sin \alpha+P_{2} \cos \alpha,
\end{gathered}
$$

где $\alpha$ - некоторая величина. После подстановки этих выражений в полином $F_{5}$ получим, что коэффициенты $A_{1}$ и $A_{3}$ при $P_{1}^{4} P_{2}$ и $P_{1}^{2} P_{2}^{3}$ имеют вид

$$
\begin{aligned}
A_{1}=\sin \alpha\left(\left(5 a_{0}-2 a_{2}\right) \cos ^{4} \alpha\right. & +\left(3 a_{2}-4 a_{4}\right) \cos ^{2} \alpha \sin ^{2} \alpha \\
& \left.+5 a_{5} \cos \alpha \sin ^{3} \alpha+a_{4} \sin ^{4} \alpha\right), \\
A_{3}=\sin \alpha\left(\left(3 a_{2}-4 a_{1}\right) \cos ^{4} \alpha\right. & +10 a_{5} \cos ^{3} \alpha \sin \alpha \\
+ & \left.2\left(5 a_{0}-3 a_{2}+3 a_{4}\right) \cos ^{2} \alpha \sin ^{2} \alpha+a_{2} \sin ^{4} \alpha\right) .
\end{aligned}
$$

Выберем $\alpha$ так, чтобы какая-то другая спектральная прямая стала вертикальной. Тогда имеют место следующие уравнения для $\alpha\left(A_{1}=A_{3}=0\right)$ :

$$
\begin{gathered}
\left(5 a_{0}-2 a_{2}\right) z^{4}+\left(3 a_{2}-4 a_{4}\right) z^{2}+5 a_{5} z+a_{4}=0, \\
\left(3 a_{2}-4 a_{1}\right) z^{4}+10 a_{5} z^{3}+2\left(5 a_{0}-3 a_{2}+3 a_{4}\right) z^{2}+a_{2}=0,
\end{gathered}
$$

где $z=\operatorname{ctg} \alpha$.

Согласно теореме 1.1 эта система принимает вид

$$
\begin{gathered}
\left(15 a_{0}+2 a_{4}\right) z^{4}-\left(5 a_{0}+7 a_{4}\right) z^{2}+a_{4}=0, \\
\left(15 a_{0}+7 a_{4}\right) z^{4}-4\left(10 a_{0}+3 a_{4}\right) z^{2}+5 a_{0}=0 .
\end{gathered}
$$

При этом система (3.3), (3.4) должна иметь два различных вещественных корня. Заметим, что $15 a_{0}+2 a_{4}$ не может быть равно нулю, так как из (3.3) следует, что $z^{2}=1 / 5$, а из (3.4) получаем

$$
15 z^{4}-20 z^{2}+1=0
$$

что невозможно. Таким образом, из (3.3) получаем

$$
z^{4}=\frac{15 a_{0} z^{2}+a_{4}\left(7 z^{2}-1\right)}{15 a_{0}+2 a_{4}} .
$$

Подставим это выражение в (3.4). Получим

$$
\frac{5\left(5 a_{0}-a_{4}\right)\left(3 a_{0}+a_{4}\right)\left(5 z^{2}-1\right)}{15 a_{0}+2 a_{4}}=0 .
$$

Отметим, что $z^{2} \neq 1 / 5$, так как из (3.3) следует, что $15 a_{0}+2 a_{4}=0$. Этот случай уже рассмотрен. При $a_{4}=5 a_{0}$ система $(3.3),(3.4)$ имеет следующие решения:

$$
z= \pm \sqrt{1-\frac{2}{\sqrt{5}}}, \quad z= \pm \sqrt{1+\frac{2}{\sqrt{5}}} .
$$

При $a_{4}=-3 a_{0}$ система $(3.3)$, (3.4) имеет решения

$$
z= \pm i, \quad z= \pm \sqrt{\frac{1}{3}} .
$$


Таким образом во всех случаях $z$ - это либо иррациональное число, либо комплексное число, но в случае решетки $\Lambda=\mathbb{Z}^{2}$ величина $z=\operatorname{ctg} \alpha$ должна быть рациональным числом. Таким образом теорема 1.2 доказана.

\section{Список литературы}

1. М.Л. Бялый, "О полиномиальных по импульсам первых интегралах для механической системы на двумерном торе", Функи. анализ и его прил., 21:4 (1987), 64-65; англ. пер.: M. L. Byalyi, "First integrals that are polynomial in momenta for a mechanical system on a two-dimensional torus", Funct. Anal. Appl., 21:4 (1987), 310-312.

2. Н. В. Денисова, В. В. Козлов, "Полиномиальные интегралы обратимых механических систем с конфигурационным пространством в виде двумерного тора", $M a$ тем. сб., 191:2 (2000), 43-63; англ. пер.: N. V. Denisova, V. V. Kozlov, "Polynomial integrals of reversible mechanical systems with a two-dimensional torus as the configuration space", Sb. Math., 191:2 (2000), 189-208.

3. В. В. Козлов, Д. В. Трещёв, "Об интегрируемости гамильтоновых систем с торическим пространством положений”, Матем. сб., 135(177):1 (1988), 119-138; англ. пер.: V.V. Kozlov, D. V. Treshchëv, "On the integrability of Hamiltonian systems with toral position space", Math. USSR-Sb., 63:1 (1989), 121-139.

4. В. В. Козлов, "Топологические препятствия к интегрируемости натуральных механических систем", Докл. АН СССР, 249:6 (1979), 1299-1302; англ. пер.: V.V. Kozlov, "Topological obstructions to the integrability of natural mechanical systems", Soviet Math. Dokl., 20:6 (1979), 1413-1415.

5. С. В. Болотин, "О первых интегралах систем с гироскопическими силами", Вестн. Моск. ун-та. Сер. 1. Матем., мех., 1984, № 6, 75-82; англ. пер.: S. V. Bolotin, "First integrals of systems with gyroscopic forces", Moscow Univ. Math. Bull., 39:6 (1984), $20-28$.

6. В.В. Козлов, Симметрии, топология и резонансы в гамилътоновой механике, Изд-во УдГУ, Ижевск, 1995; англ. пер.: V.V. Kozlov, Symmetries, topology and resonances in Hamiltonian mechanics, Ergeb. Math. Grenzgeb. (3), 31, Springer-Verlag, Berlin, 1996.

А. Е. Миронов (А. Е. Mironov)

Институт математики им. С. Л. Соболева СО РАН, г. Новосибирск;

Новосибирский государственный университет, механико-математический факультет

E-mail: mironov@math.nsc.ru
Поступило в редакцию 04.09 .2008 